\title{
A MYCOLOGICAL STUDY OF CLINICAL SAMPLES FROM SUSPECTED MYCOSES IN A TERTIARY CARE HOSPITAL
}

\author{
JITENDRA CHANDRA DEVRARI*, VARSHA SAXENA, VIDYA PAI \\ Department of Microbiology, Yenepoya Medical College, Yenepoya University, Mangalore, Karnataka, India. \\ Email: jitendradevrari87@gmail.com
}

Received: 05 December 2017, Revised and Accepted: 09 January 2018

\section{ABSTRACT}

Objective: The objective of this study was to isolate and identify the fungal agents obtained from clinical samples of suspected mycoses patients attending Yenepoya hospital.

Methods: Various clinical samples obtained from patients were subjected to preliminary examination according to standard mycological protocols such as KOH mount, wet mount, Gram's staining, and Indian ink examination. Causative organisms were identified by macroscopically and microscopically after growth on sabouraud's dextrose agar (with or without cycloheximide and chloramphenicol) and confirmed by lactophenol cotton blue mount, slide culture technique, urease test, and growth on chrome agar accordingly.

Results: Of 274 clinical samples, 125 were culture positive in which the fungal isolates obtained were dermatophytes (all 3 genera), Candida species (including Candida albicans), Fusarium species, Aspergillus fumigatus, and Cryptococcus neoformans. Male predominance was found among the patients being 6:3 male:female ratio.

Conclusion: Among the superficial infection, Trichophyton mentagrophytes was found to be predominant isolates; however, in systemic infection, C. albicans was the predominant isolates.

Keywords: Mycoses, Dermatophytes, Candida species, Aspergillus fumigatus, Fusarium species.

(C) 2018 The Authors. Published by Innovare Academic Sciences Pvt Ltd. This is an open access article under the CC BY license (http://creativecommons. org/licenses/by/4. 0/) DOI: http://dx.doi.org/10.22159/ajpcr.2018.v11i4.24127

\section{INTRODUCTION}

Fungal infections or mycoses cause a wide range of diseases in humans. Mycoses range in extent from superficial infections involving the outer layer of the stratum corneum of the skin to disseminated infection involving the brain, heart, lungs, liver, spleen, and kidneys [1]. Superficial fungal infection is the most common fungal infections. According to the World Health Organization, the prevalence rate of superficial mycotic infection worldwide has been found to be $20-25 \%$ [2]

Dermatophytes are a group of superficial fungal infections of keratinized tissues, namely, the epidermis, hair, and nails. The causative fungi are molds belonging to three asexual genera microsporum, trichophyton, and epidermophyton [3]

Systemic fungal infection in humans can range from asymptomatic pulmonary infection difficult to treat, and life-threatening disseminated infections that involve multiple organ systems [4]. Aspergillus species are frequent causes of invasive fungal infections in immunocompromised patients; they are also associated with allergic bronchopulmonary diseases, mycotic keratitis, otomycosis, and nasal sinusitis. At least 30 Aspergillus species have been associated with human diseases, and Aspergillus fumigatus remains the most frequent cause of invasive aspergillosis [5].

Invasive candidiasis (IC) includes candidemia, disseminated candidiasis with deep organ involvement, endocarditis, and meningitis. IC has an attributable mortality of $40-50 \%$ and is increasingly reported from healthcare centers. Candida albicans and non-albicans strains are both responsible for infections [6].

Objective

The objective of this study was to isolate and identify the fungal agents obtained from clinical samples of suspected mycoses patients attending Yenepoya hospital.

\section{METHODS}

This prospective study was conducted in the Department of Microbiology at Yenepoya Medical College, Mangalore, India. A total of 274 nonduplicate clinical samples were processed from December 2016 to May 2018 after obtaining ethical clearance from the institutional ethics committee.

All clinical samples (first set) were processed according to the standard protocols, i.e., (a) skin scraping was subjected to $10 \% \mathrm{KOH}$, (b) nail clippings - $40 \% \mathrm{KOH}$, (c) hairs - $20 \% \mathrm{KOH}$, (d) urine - wet mount and Gram staining, and (e) blood, sputum, bronchoalveolar lavage (BAL), and swab - Gram staining. The prepared slide was examined under the microscope for the presence of hyphae and arthrospores or yeast cells.

The second sets of samples were inoculated into sabouraud's dextrose agar slope (SDA) is used, whereas the skin scraping and nail clipping were inoculated in modified SDA (SDA with chloramphenicol and cycloheximide) [7]. The inoculation was done with a sterile chromium wire spud and center of the slope. The tubes were labeled and then incubated at room temperature. The tubes were examined every day for evidence of any growth. They were discarded as negative if there was no growth after 4 weeks. In positive cases, the growth was subcultured on to sabouraud's dextrose agar slopes without antibiotics.

The growths in tubes were seen for gross morphological characteristics, and details were recorded, namely: Rate of growth, general topography, i.e., whether flat heaped up regular or irregularly folded. Characteristics of colony, i.e., yeast like powdery granular velvety or cottony, pigmentation on the surface, and on reverse [8]. Lactophenol cotton blue mount was used in all positive growth culture to check the microscopic finding. Special tests were done when necessary, namely, germ tube test, slide culture, and urease test for species identification. 
Statistical analysis

Descriptive statistics were used for analysis. Variables were expressed as percentages. All the data were expressed as table diagrams.

\section{RESULTS}

The distribution of various types of 274 clinical samples is as follows: Skin scraping was 127 , urine 37, blood culture 34, nail clipping 19, pus 16 , sputum 9, tissue 9, CSF 6 , BAL 5 , corneal scrap 5 , nasal swab 4 , and hair 4 . In this study, male-female ratio was found to be 6:3. Of 274 clinical samples, 125 (45.6\%) showed fungal growth positive and 149 (54.4\%) sample showed no growth. Of 274 samples, 172 were male and 102 were female, as shown in Fig. 1 and Table 1.

In this study, the predominant age group in blood and urine samples was $0-10$ years, among skin scraping, nail clipping and hair samples were $20-30$ years, among pus and tissue were $30-40$ years, nasal swab and corneal scrapings were 40-50 years, sputum was 5060 years, CSF was $0-10$ years, and BAL was $60-70$ years as shown in Table 2 .

Among 126 skin scrapings, 109 samples were found to be $\mathrm{KOH}$ (10\%) positive and 17 were negative, whereas only 87 (69\%) were $\mathrm{KOH}$ as well as culture positive in which Trichophyton mentagrophytes was the predominant isolate. All 37 urine samples were subjected to wet mount and culture, in which only 14 (37.8\%) samples were positive for both, whereas C. albicans was isolated from 9 samples and $C$. tropicalis was isolated from 5 samples. Among 34 blood culture samples, only $2(\%)$ were found to be BACTEC and culture positive for $C$. albicans. Among 19 nail clipping samples, 6 were $\mathrm{KOH}(40 \%)$ positive and $9(47.4 \%)$ were both $\mathrm{KOH}$ and culture positive; in which 3 were Trichophyton species, 3 were Fusarium species, and 3 were $C$. albicans. Of 16 pus samples, only $1(6.3 \%)$ was culture positive. Of 9 sputum, 5 BAL and 5 corneal scrapings samples; 5 (55.5\%), 1 (20\%), and 1 (20\%) were culture positive respectively in which Candida species (including $C$. albicans) was the only isolate. Among 9 tissue samples only 2 (22.2\%) samples

Table 1: Distribution of fungus samples

\begin{tabular}{lllll}
\hline Sample & Male & Female & $\begin{array}{l}\text { Total samples } \\
(\mathbf{n = 2 7 4 )}\end{array}$ & $\begin{array}{l}\text { Culture } \\
\text { positive (\%) }\end{array}$ \\
\hline Skin scraping & 76 & 50 & 126 & $87(69)$ \\
Urine & 19 & 18 & 37 & $14(37.8)$ \\
Blood culture & 22 & 12 & 34 & $2(5.9)$ \\
Nail clipping & 11 & 8 & 19 & $9(47.4)$ \\
Pus & 12 & 4 & 16 & $1(6.3)$ \\
Sputum & 7 & 2 & 09 & $5(55.5)$ \\
Tissue & 8 & 1 & 09 & $2(22.2)$ \\
CSF & 5 & 1 & 06 & $1(16.7)$ \\
BAL & 4 & 1 & 05 & $1(20)$ \\
Corneal scrape & 4 & 1 & 05 & $1(20)$ \\
Nasal swab & 2 & 2 & 04 & $2(50)$ \\
Hair & 2 & 2 & 04 & $0(0)$ \\
Total & 172 & 102 & 274 & 125 \\
\hline
\end{tabular}

BAL: Bronchoalveolar lavage were $\mathrm{KOH}$ and culture positive; in which one was $C$. albicans and one was Fonsecaea pedrosoi. Among 4 nasal swabs, only $2(50 \%)$ showed growth of Aspergillus flavus. Among 6 CSF samples, only 1 (16.7\%) was Indian ink and culture positive for Cryptococcus neoformans. However, among 4 hair samples, none of them were $\mathrm{KOH}(20 \%)$ as well as culture positive as shown in Tables 1 and 3; and macroscopic and microscopic appearance as shown in Figs. 2 and 3.

\section{DISCUSSION}

In the present study, of 274 clinical samples, 125 were culture positive in which the fungal isolates obtained were dermatophytes (all 3 genera), Candida species (including C. albicans), Fusarium species, A. fumigatus, and C. neoformans.

Among superficial fungal infections, most frequent isolates belonged to the genera Trichophyton in which T. mentagrophytes was found to be predominant species followed by Microsporum gypseum, M. canis, and Fusarium species. However, in case of systemic infection, Candida species (including C. albicans) was predominant isolate followed by A. fumigatus, whereas only one $C$. neoformans has been grown out of 6 CSF samples. Male predominance was found among the patients being 6:3 male:female ratio. However, our study is discordance with the other studies which have reported T. rubrum is the most common isolate from the superficial infections, whereas among systemic infections, our

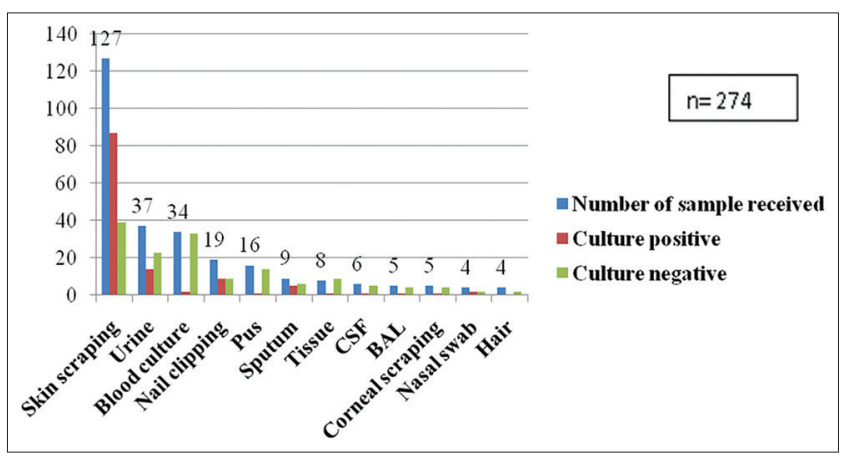

Fig. 1: Comparison between number of sample received and culture positive

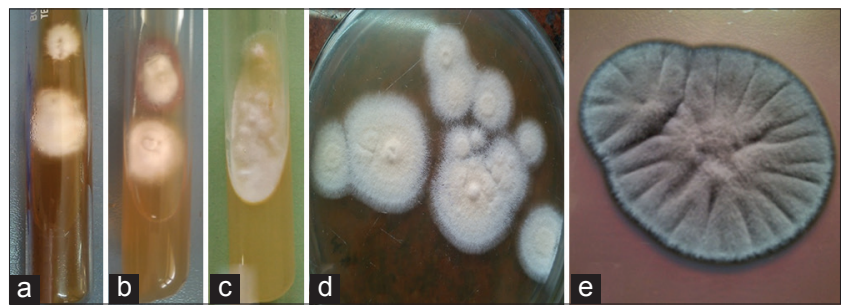

Fig. 2: Macroscopic appearance (a) Trichophyton mentagrophytes, (b) Trichophyton rubrum, (c) Epidermophyton floccosum, (d) Microsporum gypseum, (e) Fonsecaea pedrosoi

Table 2: Age wise distribution of sample

\begin{tabular}{|c|c|c|c|c|c|c|c|c|c|c|c|c|}
\hline Age group (years) & BC & BAL & SPU & PUS & NS & SS & Hair & NC & Urine & Tissue & CS & CSF \\
\hline $0-10$ & 29 & - & - & - & - & 03 & - & - & 30 & - & - & 03 \\
\hline $10-20$ & 01 & 01 & - & 01 & - & 29 & 01 & 02 & 03 & - & 01 & 01 \\
\hline $20-30$ & - & - & - & 02 & 01 & 40 & 02 & 09 & 01 & - & - & - \\
\hline $30-40$ & 01 & 01 & 01 & 04 & - & 06 & - & - & - & 06 & - & 01 \\
\hline $40-50$ & 02 & - & 02 & 03 & 03 & 20 & - & 04 & - & - & 03 & - \\
\hline $50-60$ & - & 01 & 04 & 03 & - & 23 & 01 & 02 & 01 & 02 & 01 & 01 \\
\hline $60-70$ & 01 & 02 & 02 & 02 & - & 05 & - & - & 01 & 01 & - & - \\
\hline Total & 34 & 05 & 09 & 16 & 04 & 126 & 04 & 19 & 37 & 09 & 05 & 06 \\
\hline
\end{tabular}

BC: Blood culture, BAL: Bronchoalveolar lavage, SPU: Sputum, NS: Nasal swab, SS: Skin scraping, NC: Nail clipping, CS: Corneal scraping, CSF: Cerebrospinal fluid 


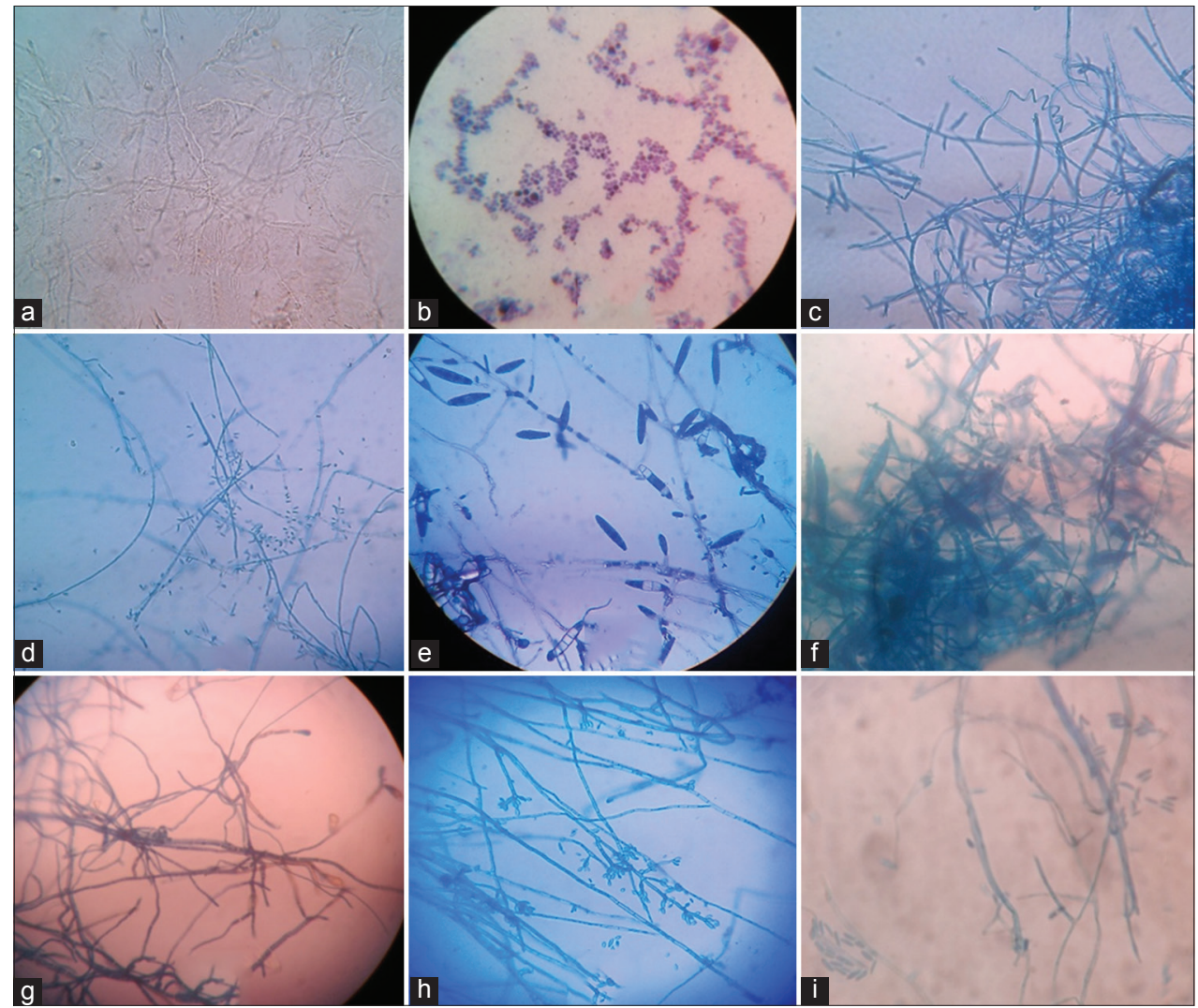

Fig. 3: Microscopic appearance (a) KOH (10\%) positive, (b) Candida species, (c) Trichophyton mentagrophytes, (d) Trichophyton rubrum, (e) Microsporum gypseum, (f) Microsporum canis, (g) Epidermophyton floccosum, (h) Fonsecaea pedrosoi, (i) Fusarium species

Table 3: Types of fungal isolated from all samples

\begin{tabular}{ll}
\hline Fungal species & Total (\%) \\
\hline Trichophyton mentagrophytes & $49(39.2)$ \\
Trichophyton rubrum & $15(12)$ \\
Trichophyton species & $09(7.2)$ \\
Microsporum gypseum & $11(8.8)$ \\
Microsporum canis & $01(0.8)$ \\
Epidermophyton floccosum & $01(0.8)$ \\
Fusarium species & $05(4)$ \\
Aspergillus fumigatus & $02(1.6)$ \\
Candida albicans & $24(19.2)$ \\
Candida tropicalis & $06(4.8)$ \\
Cryptococcus neoformans & $01(0.8)$ \\
Fonsecaea pedrosoi & $01(0.8)$ \\
Total & $125(100)$ \\
\hline
\end{tabular}

study is in accordance with the other studies in which Candida species being the most common pathogen.

In this study, among children (0-10 years), the most common samples received were blood and urine, whereas among adults (20-30 years), the most common sample received was skin scrapings.

In accordance with our study, a study was conducted by Malik et al. [9] also showed the higher incidence of fungal infection mainly dermatophytes in males than in females has been reported in India. Among dermatophytoses, total 86 isolates were obtained from the all skin scraping and nail clippings. The dermatophytes isolated were T. mentagrophytes, $T$. rubrum, M. gypseum, M. canis, and Epidermophyton floccosum. Candida spp. were isolated from 30 cases, of which 26 (19.4\%) were C. albicans and $6(4.8 \%)$ were $C$. tropicalis. However, according to Kannan et al. [10] showed the higher prevalence of T. rubrum among cases of dermatophytoses, whereas Candida species being the predominant isolate from all non-dermatophytic fungal infections. Kuruvilla and Dias [11] and Jandial and Shaveta [12] reported that Fusarium species from dermatophytoses samples as similar to our study, whereas Malik et al. reported higher prevalence as compared to the present study. However, in the same study, by Malik et al., A. fumigatus was $15.6 \%$ which is much higher than the present study $(1.6 \%)$. In the present study, Fonsecaea pedrosoi was detected from one out of two tissue sample. A similar result was also reported in case report done by Khan et al. from Kochi [13].

\section{CONCLUSION}

The frequency of superficial and systemic mycoses has increased significantly. This increase infection is mostly because of underdiagnosis of the causative pathogen and is directly related to the increasing numbers of patients who are at risk for the development of serious fungal infections. Therefore, all the fungal infections should be dealt with due care. The development of new approaches to diagnosis and treatment of invasive fungal infections is the subject of intensive research.

\section{AUTHOR'S CONTRIBUTION}

Jitendra Chandra Devrari and Varsha Saxena have contributed to the study conception, design, sample collection, analysis, and interpretation of the data and manuscript preparation. Vidya Pai has contributed to the conception and critical revision of the manuscript.

\section{CONFLICT OF INTEREST}

None.

\section{REFERENCES}

1. Baron S, editor. Medical Microbiology. $4^{\text {th }}$ ed. Galveston (TX): University of Texas Medical Branch at Galveston; 1996. 
2. Lakshmanan A, Ganeshkumar P, Mohan SR, Hemamalini M, Madhavan R. Epidemiological and clinical pattern of dermatomycoses in rural India. Indian J Med Microbiol 2015;33:134-6.

3. Tonita M, Noronha TM, Tophakhane RS, Nadiger S. Clinico-microbiological study of dermatophytosis in a tertiary care hospital in North Karnataka. Indian Dermatol Online J 2016;7:264-71.

4. Ray A, Anand S. Recent trends in antifungal therapy: Focus on systemic mycoses. Indian J Chest Disease Allied Sci 2000;42:357-66.

5. Diba K, Kordbacheh P, Mirhendi SH, Rezaie S, Mahmoudi M. Identification of Aspergillus species using morphological characteristics. Pak J Med Sci 2007;23:867-72.

6. De Rosa FG, Garazzino S, Pasero D, Di Perri G, Ranieri VM. Invasive candidiasis and candidemia: New guidelines. Minerva Anestesiol 2009;75:453-8.

7. Kak S, Bhat RM, Boloor R, Nandakishore B, Sukumar DA. Clinical and mycological study of dermatophytic infections. Indian J Dermatol 2014;59:262-7.
8. Pai VV, Hanumanthayya K, Kikkeri NC. Clinical study of tinea capitis in Northern Karnataka: A three-year experience at a single institute. Indian Dermatol Online J 2013;4:22-6.

9. Malik A, Fatima N, Khan PA. A clinico-mycological study of superficial mycoses from a tertiary care hospital of a North Indian town. Virol Mycol 2014;3:135-8.

10. Kannan P, Janaki C, Selvi GS. Prevalence of dermatophytes and other fungal agents isolated from clinical samples. Indian J Med Microbiol $2006 ; 24: 212-5$.

11. Kuruvilla TS, Dias M. Fusarium solani: A causative agent of skin and nail infections. Indian J Dermatol 2012;57:308-9.

12. Jandial S, Sumbali G. Fusarial onychomycosis among gardeners: A report of two cases. Indian $\mathrm{J}$ Dermatol Venereol Leprol 2012;78:229- 30

13. Khan S, Kumar A, Vinod B, Prabhakar V, Eapen M, Thomas J, et al. Chromoblastomycosis due to Fonsecaea pedrosoi: An old wine in a rare bottle. J Infect Dev Ctries 2015;9:325-9. 